\title{
Usefulness of Gd-EOB-DTPA Liver Dynamic MRI for Diagnosing Peliosis Hepatis Mimicking a Solitary Hepatic Mass with Central Necrosis: A
}

\section{Case Report}

\author{
Hyun Kyung Lim, ${ }^{1}$ Hong Il Ha, ${ }^{2,}{ }^{*}$ and Kyueng-Whan Min ${ }^{2}$ \\ ${ }^{1}$ Department of Radiology, Soonchunhyang University College of Medicine, Soonchunhyang University Seoul Hospital, Seoul, Republic of Korea \\ ${ }^{2}$ Department of Radiology, Hallym University Medical Center, Hallym Sacred Heart Hospital, Gyeonggi-do, Republic of Korea \\ "Corresponding author: Hong Il Ha, Department of Radiology, Hallym University Medical Center, Hallym Sacred Heart Hospital 22, Gwanpyeong-ro 170beon-gil, Dongan-gu, \\ Anyang-si, Gyeonggi-do, 14068, Republic of Korea. Tel: +82-313803880, Fax: +82-313803880, E-mail: ha.hongil@gmail.com \\ Received 2016 January 06; Revised 2016 February 23; Accepted 2016 March 29.
}

\begin{abstract}
Peliosis hepatis ( $\mathrm{PH})$ is a rare benign disease of the liver and is characterized by the dilatation of the sinusoids of the liver parenchyma. Here, we present a rare case of PH presenting as a solitary mass with a large central cystic change mimicking a solitary hepatic malignant neoplasm. In addition, we review the literature, focusing on the particular usefulness of the Gd-EOB-DTPA hepatocyte-specific magnetic resonance imaging contrast agent.
\end{abstract}

Keywords: Peliosis Hepatis, Liver, Gadolinium Ethoxybenzyl DTPA, Magnetic Resonance Imaging

\section{Introduction}

Peliosis hepatis $(\mathrm{PH})$ is a rare benign disease that is detected in liver imaging (1). It is characterized by the dilatation of the hepatic sinusoids presenting as variablesized, blood-filled cystic spaces on pathology. Although the exact pathogenesis of $\mathrm{PH}$ is unknown, it has been associated with malignancies, immunosuppression, chronic wasting disease, infection, and certain medications such as anabolic steroids, corticosteroids, or oral contraceptives (1, 2 ). It is difficult to diagnose $\mathrm{PH}$, because its imaging features are non-specific and can appear similar to malignant tumors, metastasis, or abscesses (3-6). Here, we present a case of a large $\mathrm{PH}$ in a serious alcoholic patient who was hepatitis $\mathrm{B}$ antigen-positive. The patient was examined by liver dynamic magnetic resonance imaging (MRI) using the Gadolinium ethoxybenzyl diethylenetriamine pentaacetic acid (Gd-EOB-DTPA) hepatocyte-specific MRI contrast agent. We also review the literature on $\mathrm{PH}$ in relation to its pathology and the imaging findings of Gd-EOB-DTPA liver MRI.

\section{Case Presentation}

A 57-year-old male was referred to our institution by a local clinic due to uncontrolled type II diabetes mellitus and a large hepatic mass detected incidentally on ultrasonography (US). The patient had experienced an 18-kg weight loss during the previous 3 months as well as general weakness. A physical examination revealed no spe- cific abnormality. Laboratory testing showed a mild elevation of both aspartate transaminase (43 IU/L) and alanine transaminase ( $51 \mathrm{IU} / \mathrm{L})$. The patient's fasting blood glucose level was $518 \mathrm{mg} / \mathrm{dL}$, and his glycated hemoglobin (Hb A1c) level was $14.6 \%$. The hepatitis B antigen was determined as positive, but the tumor marker alfa fetoprotein was within the normal range.

On a transabdominal US examination at our hospital, an ill-defined hyperechoic mass of approximate size 6.5 $\mathrm{cm}$ was detected in hepatic segment IV. It had a central irregular anechoic lesion, and a color Doppler US indicated no increased blood flow to the lesion (Figure $1 \mathrm{~A}$ and $1 \mathrm{~B}$ ). A four-phase liver dynamic multidetector computed tomography (MDCT) scan was then performed. The mass was located at the dome of hepatic segment IV between the middle and left hepatic veins. However, there was no observed mass effect caused by the mass. On pre-contrast phase imaging, the mass showed a triple-density-layered pattern consisting of a central low density area surrounded by a middle thick isodensity layer adjacent to the normal liver parenchyma that was engulfed by a low attenuation outer layer, which suggested perilesional edema (Figure $2 \mathrm{~A}$ ). The middle thick layer that was found to be isodense on precontrast CT was enhanced persistently and strongly during the arterial to delayed phase (Figure $2 \mathrm{~B}-2 \mathrm{D}$ ). The outer low density layer, which suggested perilesional edema, showed as isodense on the 3 minutes delayed phase (Figure 2D). An MRI was then performed using dynamic imaging, including imaging in the arterial phase (30 seconds), por- 
tal venous phase (60 seconds), and transitional phase (3 minutes), in addition to the hepatobiliary phase (20 minutes), after administration of $0.1 \mathrm{mmol} / \mathrm{kg}$ Gd-EOB-DTPA (Primovist®; Bayer Healthcare, Berlin, Germany). The mass showed a low-signal intensity on T1-weighted imaging (Figure 3A). On T2-weighted half-Fourier acquisition turbo spin-echo images, the mass had triple signal intensities such that the central lesion was strongly hyperintense, similar to the signal intensity of cerebrospinal fluid, the middle thick layer was isointense to intermediate hyperintense, and the outer layer was hyperintense (Figure 3B). The middle thick layer of the lesion was persistently strongly enhanced from the arterial to the transitional phase (Figure $3 \mathrm{C}-3 \mathrm{E}$ ). On hepatobiliary phase imaging, the lesion was still visible, but showed less intense enhancement compared with the adjacent normal liver parenchyma (Figure $3 F$ ). The middle thick irregular layer showed a decreased value for the apparent diffusion coefficient (ADC), and the central portion showed a high ADC value (Figure 3G). Under US guidance, several target biopsies were performed on both lateral sides of the lesion, and the lesion was finally diagnosed as PH (Figure 4). This patient was treated with a broad spectrum cephalosporin antibiotic (Cefotaxime) for parenteral administration. After 2 month follow up, $\mathrm{PH}$ had been reduced from $6.5 \mathrm{~cm}$ to $3.5 \mathrm{~cm}$ (Figure 2E).

\section{Discussion}

$\mathrm{PH}$ is a rare benign disorder characterized by dilatation of the hepatic sinusoids and the presence, along with the hepatic parenchyma, of blood-filled lacunar spaces of various sizes. Although the exact pathogenesis of PH remains unclear, it may result from necrosis of the hepatocytes and a blockage in the hepatic blood outflow at the junction of the sinusoids and centrilobular veins (2).

$\mathrm{PH}$ is frequently related to various conditions, such as chronic wasting disease, tuberculosis, malignancies, immunosuppression, diabetes, and chronic alcoholism as well as certain medications such as anabolic steroids, corticosteroids, tamoxifen, or oral contraceptives. Our patient had uncontrolled type II diabetes and chronic severe alcoholism $(1,2)$.

The diagnosis of $\mathrm{PH}$ is difficult because the disease lacks specific radiologic findings $(4,5,7)$. On sonography, the echo pattern of $\mathrm{PH}$ varies and is inhomogeneous (5). MDCT and MRI are helpful for detecting and diagnosing PH. On pre-contrast CT scans, PH was found to have a low density or isodensity compared with the liver parenchyma. After bolus administration of a contrast material, $\mathrm{PH}$ showed a gradual enhancement from the arterial to delayed phases. The lesions may show as a low density area or as central enhanced foci on arterial phase and become more prominently enhanced on portal and delayed phase scans. Centrifugal pattern enhancement and the absence of a mass effect are characteristic imaging findings of $\mathrm{PH}$ (3-5). However, PH presenting as a solitary mass is very rare, and only a few case reports have been published to date. A large $\mathrm{PH}$ with a prominent central cystic change like our case has not, to our knowledge, been previously reported, and this makes our case more difficult to differentiate from other malignant tumors or abscesses. The PH in our case showed intense enhancement from the arterial phase, and this enhancement was prolonged into the transitional and hepatobiliary phases. There was also no mass effect, even though the lesion was large and abutted into the middle hepatic vein. These imaging findings were similar to previously established $\mathrm{PH}$ imaging features (3-5). The large central cystic lesion was thought to be necrosis of a malignant tumor or pus from an abscess. However, it was finally considered to be a hemorrhage resulting from the destruction of the hepatic sinusoids, probably caused by a massive hemorrhage due to the rupture of the $\mathrm{PH}(8,9)$. These imaging features, especially in MR imaging features using the extracellular contrast agent, are nonspecific and similar as other hypervascular hepatic tumors.

Gd-EOB-DTPA has unique characteristic pharmacodynamics during MRI imaging. In the vascular phase, this agent acts as a conventional nonspecific extracellular contrast agent. In the delayed phase at around $20 \mathrm{~min}$, this agent is actively transported from the sinusoidal space into hepatocytes via organic anion transporting polypeptides and causing intense hepatic parenchymal enhancement (10). By this mechanism, uptake by functioning hepatocytes in the delayed phase provides additional information for detection and characterization of various hepatic lesions (11). Among the various hepatic lesions, $\mathrm{PH}$ should be differentiated from regenerative or dysplastic nodule, adenoma, hemangioma and focal nodular hyperplasia (FNH) because these lesions show persistent enhancement during the dynamic phase or isointensity in the hepatobiliary phase. Regenerative or dysplastic nodule is commonly encountered in the cirrhotic liver. This lesion is usually isosignal intensity in the hepatobiliary phase and this lesion does not show intense enhancement beyond arterial or portal phases. Adenoma is one of hypervascular tumor thus it shows well enhancement in the arterial phase and isosignal intensity in the portal phase. However, adenoma shows defect in the hepatobiliary phase. Hemangioma is common benign hypervascular mass and its signal intensity on the dynamic MR image is similar as the enhancing vascular signal intensity. But, hemangioma shows pseudo-washout in the hepatobiliary phase. FNH is the second most common benign hepatic tumor. It con- 

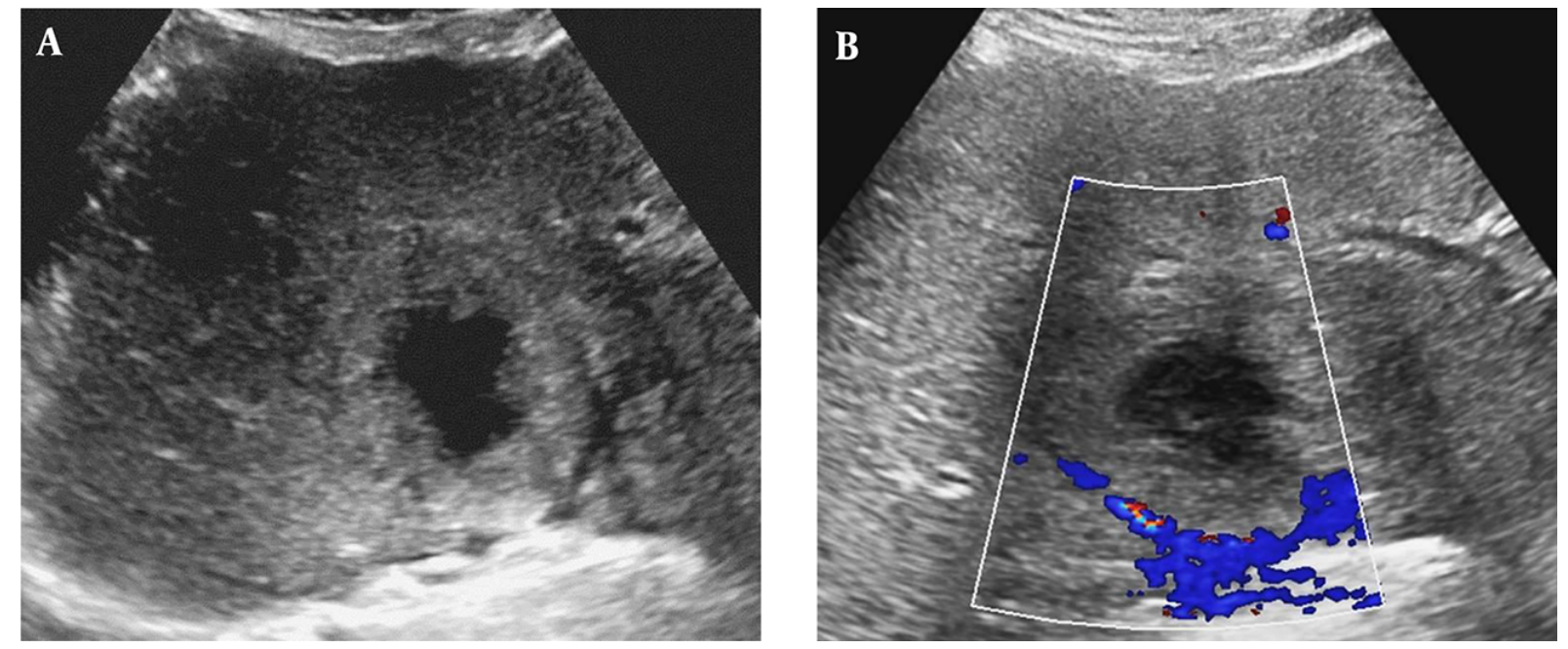

Figure 1. Ultrasonography (US) of a 57-year-old man with a $6.5 \mathrm{~cm}$ solitary peliosis hepatis undergoing a large central cystic change. A, Grayscale transabdominal US shows an ill-defined hyperechoic lesion in S4 of the liver. An irregular large anechoic cystic content is noted in the central portion of the lesion; B, Color Doppler US shows no increased vascularity of the lesion.

sists of aggregates of hepatocytes and biliary structures without connection to the adjacent biliary tree. FNH shows marked uniform enhancement in the arterial phase. This enhancement is lessening but persistent during portal and delayed phases, the lesion signal intensity is similar as that of the surrounding hepatic parenchyma. FNH represents as isointense or hyperintense to the normal liver in the hepatobiliary phase. Malignant tumors such as hepatocellular carcinoma, cholangiocarcinoma or metastasis show variable enhancement pattern in the dynamic MR image, however, these lesions represent as hypointense to the normal hepatic parenchyma in the hepatobiliary phase (11). $\mathrm{PH}$ consists of normal hepatocytes and dilated sinusoids (2). Theoretically, it will take up the Gd-EOB-DTPA contrast agent and present as a hyperintense lesion on hepatobiliary phase imaging. Kim et al. reported hyperintense HCC on hepatobiliary phase images of Gd-EOB-DTPA MRI and noted that the change in peliosis around the HCC was significant on pathologic analysis (12).

The PH in our case showed strong persistent enhancement from arterial to portal phase, similar as other studies using conventional extracellular contrast agent (11). This enhancement had been maintained to the hepatobiliary phase. Persistent delay enhancement of $\mathrm{PH}$ in our case is similar with the enhancement pattern of $\mathrm{FNH}$, because both FNH and $\mathrm{PH}$ is consist of the normal hepatocytes. The $\mathrm{PH}$ in our case showed persistent, but reduced, enhancement compared with the adjacent liver parenchyma on hepatobiliary phase. We considered that relatively low density of hepatocyte caused by sinusoidal dilatation in $\mathrm{PH}$ might be cause of relatively less enhancement of the $\mathrm{PH}$ compared with the adjacent normal liver parenchyma on hepatobiliary phase imaging. Thus, we suggest that this persistent enhancement feature of $\mathrm{PH}$ on hepatobiliary phase imaging should be considered as an important differential point because this imaging feature results from normal functioning hepatocytes, in addition to the characteristic centrifugal enhancement pattern and lack of a mass effect.

In conclusion, we reported a rare case of a large solitary $\mathrm{PH}$ with a large central cystic area, which showed persistent enhancement on Gd-EOB-DTPA MRI. Persistent enhancement on hepatobiliary phase imaging as well as ongoing intense enhancement during the dynamic phase, in addition to the absence of a mass effect, are considered to be the characteristic imaging features of $\mathrm{PH}$.

\section{Acknowledgments}

This work was supported by the Soonchunhyang university research fund.

\section{Footnotes}

Authors' Contribution: Hyun Kyung Lim wrote the manuscript. The manuscript was edited under the supervision of Hong Il Ha. Kyueng-Whan Min gave advice on the pathology aspects of this case.

Financial Disclosure: The authors have nothing to disclose. 

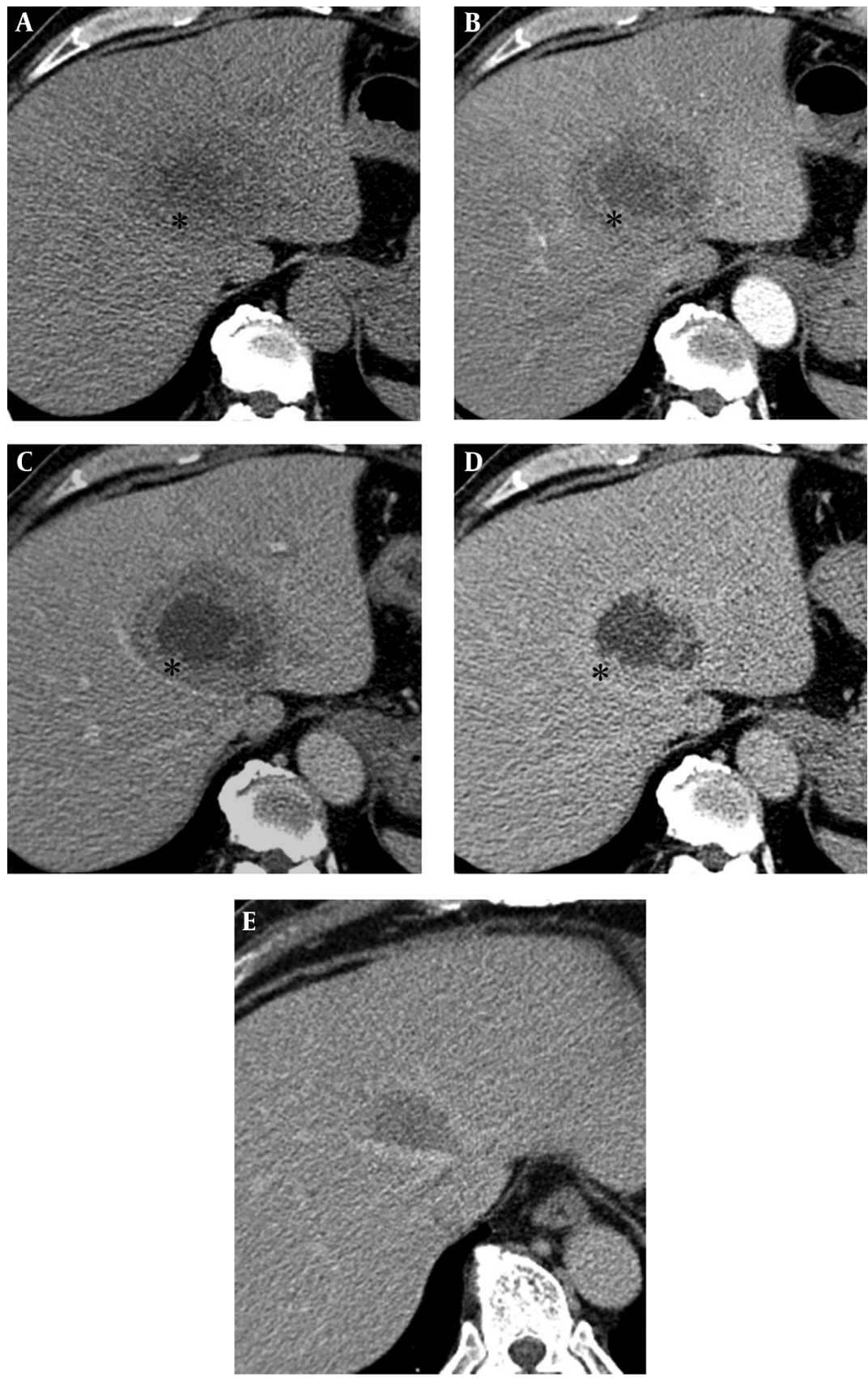

Figure 2. Multidetector computed tomography (CT) of a 57-year-old man with a $6.5 \mathrm{~cm}$ solitary peliosis hepatis undergoing a large central cystic change. A, A pre-contrast axial CT scan shows an ill-defined lesion with a triple layered appearance. The central portion is of low density, and the middle irregular thick layer $\left({ }^{*}\right)$ shows as isodense compared with the adjacent liver parenchyma, while the outer layer shows a hypodense rim similar to perilesional edema; B, C, After contrast medium enhancement, the middle irregular thick layer $\left({ }^{*}\right)$ shows strong enhancement from the arterial to delayed phase; D, A perilesional low density halo disappeared at the delayed phase. The central low density area does not show any enhancement; E, PH has been decreased in size from $6.5 \mathrm{~cm}$ to $3.5 \mathrm{~cm} 2$ months after conservative treatment with antibiotics. 

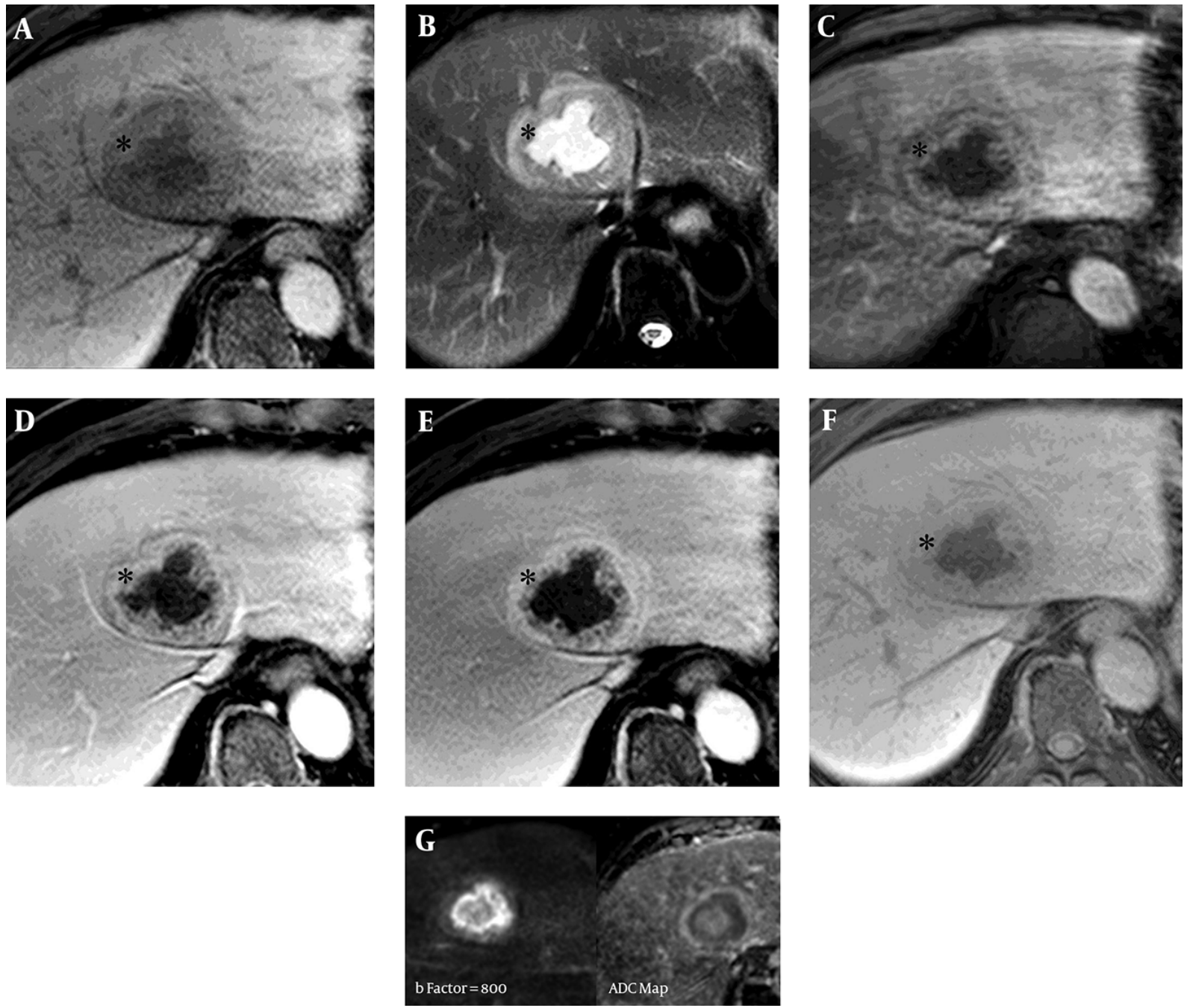

Figure 3. Liver magnetic resonance imaging (MRI) of a 57 -year-old man with a $6.5 \mathrm{~cm}$ solitary peliosis hepatis undergoing a large central cystic change. A, Liver MRI was performed using dynamic imaging including imaging in the arterial phase (C, 30 seconds), portal venous phase ( $\mathrm{D}, 60$ seconds), and transitional phase (, 3 minutes), as well as the hepatobiliary phase (F, 20 minutes), after administration of $0.1 \mathrm{mmol} / \mathrm{kg} \mathrm{Gd-EOB-DTPA.} \mathrm{The} \mathrm{lesion} \mathrm{is} \mathrm{hypointense} \mathrm{on} \mathrm{pre-contrast} \mathrm{T1-weighted} \mathrm{imaging;} \mathrm{B,} \mathrm{On} \mathrm{T2-weighted}$ half-Fourier acquisition turbo spin-echo images, it shows a triple signal intensity. The central area shows as hyperintense, similar to cerebrospinal fluid, and the thick middle layer ${ }^{*}$ ) shows iso- to slight hyperintensity compared with the adjacent liver parenchyma; C - E, The peripheral area shows as a hyperintense halo. In comparison with the CT scans, the thick middle layer ${ }^{*}$ ) surrounding the central cystic lesion shows more intense enhancement compared with the adjacent liver parenchyma from the arterial to the transitional phase (3 minutes); F, On hepatobiliary phase, the thick middle layer ${ }^{*}$ ) has been enhanced, but less strongly than the adjacent liver parenchyma; G, The enhancing peripheral lesion shows decreased diffusivity on diffusion weighted image (b factor, 800) and ADC map (ADC, $\left.1.04 \times 10^{-3} \mathrm{~mm}^{2} / \mathrm{s}\right)$. 


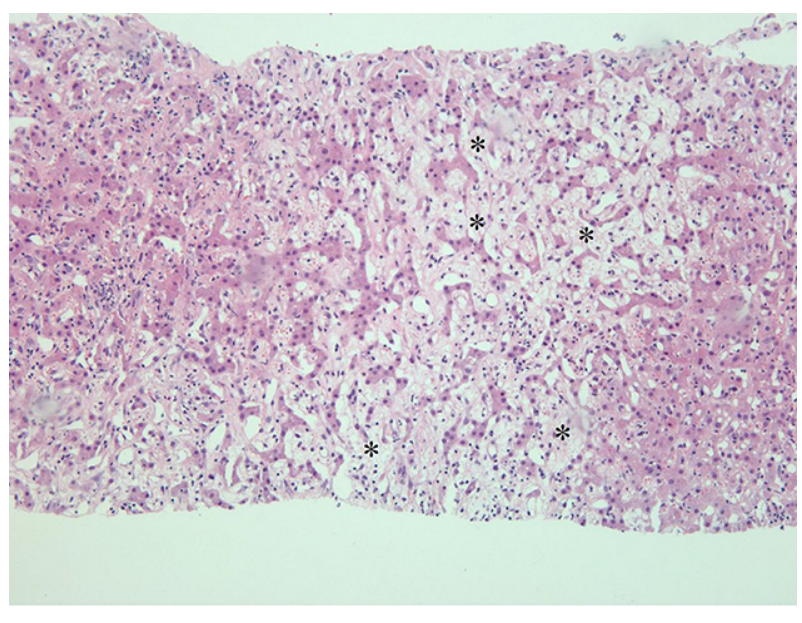

Figure 4. Photomicroscopic image shows dilatation of the sinusoid $\left(^{*}\right)$ (hematoxylin and eosin, $\times 100)$.

Funding/Support: This work was supported by the Soonchunhyang university research fund. This fund was used to prepare the manuscript editing and publish.

\section{References}

1. Torabi M, Hosseinzadeh K, Federle MP. CT of nonneoplastic hepatic vascular and perfusion disorders. Radiographics. 2008;28(7):1967-82. doi: 10.1148/rg.287085067. [PubMed:19001652].

2. Zafrani ES, Cazier A, Baudelot AM, Feldmann G. Ultrastructural lesions of the liver in human peliosis. A report of 12 cases. Am J Pathol. 1984;114(3):349-59. [PubMed: 6696047].
3. Gouya H, Vignaux O, Legmann P, de Pigneux G, Bonnin A. Peliosis hepatis: triphasic helical CT and dynamic MRI findings. Abdom Imaging. 2001;26(5):507-9. [PubMed: 11503089].

4. Iannaccone R, Federle MP, Brancatelli G, Matsui O, Fishman EK, Narra VR, et al. Peliosis hepatis: spectrum of imaging findings. AJR Am J Roentgenol. 2006;187(1):43-52. doi: 10.2214/AJR.05.0167. [PubMed: 16794138].

5. Kim SH, Lee JM, Kim WH, Han JK, Lee JY, Choi BI. Focal peliosis hepatis as a mimicker of hepatic tumors: radiologicalpathological correlation.J Comput Assist Tomogr. 2007;31(1):79-85. doi: 10.1097/01.rct.0000232919.22287.20. [PubMed: 17259837].

6. Xiong WJ, Hu LJ, Jian YC, He Y, Zhou W, Guo XL, et al. Focal peliosis hepatis in a colon cancer patient resembling metastatic liver tumor. World J Gastroenterol. 2012;18(41):5999-6002. doi: 10.3748/wjg.v18.i41.5999. [PubMed: 23139621].

7. Crocetti D, Palmieri A, Pedulla G, Pasta V, D’Orazi V, Grazi GL. Peliosis hepatis: Personal experience and literature review. World J Gastroenterol. 2015;21(46):13188-94. doi: 10.3748/wjg.v21.i46.13188. [PubMed: 26675327].

8. Kim EA, Yoon KH, Jeon SJ, Cai QY, Lee YW, Yoon SE, et al. Peliosis hepatis with hemorrhagic necrosis and rupture: a case report with emphasis on the multi-detector CT findings. Korean J Radiol. 2007;8(1):64-9. doi: 10.3348/kjr.2007.8.1.64. [PubMed: 17277565].

9. Vignaux O, Legmann P, de Pinieux G, Chaussade S, Spaulding C, Couturier D, et al. Hemorrhagic necrosis due to peliosis hepatis: imaging findings and pathological correlation. EurRadiol. 1999;9(3):454-6. doi: 10.1007/s003300050691. [PubMed: 10087115].

10. Tsuboyama T, Onishi H, Kim T, Akita H, Hori M, Tatsumi M, et al. Hepatocellular carcinoma: hepatocyte-selective enhancement at gadoxetic acid-enhanced MR imaging-correlation with expression of sinusoidal and canalicular transporters and bile accumulation. Radiology. 2010;255(3):824-33. doi: 10.1148/radiol.10091557. [PubMed: 20501720].

11. Goodwin MD, Dobson JE, Sirlin CB, Lim BG, Stella DL. Diagnostic challenges and pitfalls in MR imaging with hepatocyte-specific contrast agents. Radiographics. 2011;31(6):1547-68. doi: 10.1148/rg.316115528. [PubMed: 21997981].

12. Kim JY, Kim MJ, Kim KA, Jeong HT, Park YN. Hyperintense HCC on hepatobiliary phase images of gadoxetic acid-enhanced MRI: correlation with clinical and pathological features. Eur J Radiol. 2012;81(12):387782. doi:10.1016/j.ejrad.2012.07.021. [PubMed: 22954410]. 